Polyether ether ketone (PEEK), a material commonly used in industry, has recently been introduced in medicine. The use of PEEK in place of gold in gold marker implantation for prostate radiotherapy has been suggested to be advantageous because it is associated with a lower level of artefacts. In this study, we present a disadvantage of this newly introduced method, which has not yet been defined in the literature, and offer a solution for this problem to shed light on this issue, which has not yet been completely understood.

Key words: PEEK markers, fiducial markers, prostate cancer.

Contemp Oncol (Pozn) 2018; 22 (1): 47-49 DOI: https://doi.org/10.5114/wo.2018.74394

\section{Our experiences and suggestions with PEEK marker use in prostate cancer radiotherapy}

\author{
Cenk Ahmet Sen
}

Department of Radiation Oncology, Izmir Medical Park Hospital, Izmir, Turkey

PEEK is a semi-crystal and high-degree pure polymer composed of two ethers and one repetitive monomer in the ketone group. It demonstrates all the properties of durability expected from a thermoplastic material. It shows high durability against chemical and mechanical effects and heat. It has complete recyclability. It was first produced in 1982 and has been widely used in the industry since then. Currently it is used in medicine thanks to its various advantageous properties. It is engineered for strength, it has stability and biocompatibility, is resistant against radiation, it is compatible with magnetic resonance imaging (MRI), it causes minimal MRI and computed tomography (CT) artefacts, and it has bone-like stiffness, strength, and toughness, and is easily sterilised. It has been also used in neurosurgery, cardiovascular surgery, and dentistry in current practice. The types of material used in those areas are classified as mentioned below [1].

Clinical classification of PEEK implants:

- PEEK for bone replacement-maxillofacial and cranial implants,

- PEEK for spine surgery-spinal cages,

- PEEK for orthopaedic surgery; for bone and hip-replacement-articulation implants and orthopaedic devices from PEEK material-fixation plates and screws,

- PEEK for tooth replacement-dental implants; dental prosthesis, intra-radicular posts,

- PEEK for cardiac surgery-intracardiac pump; heart valves.

Recently, reports have been published on the use of PEEK materials in RT. The contribution of PEEK implants has been shown in a study by Nevelsky et al. [2] to overcome the uncertainty in dose distribution caused by the metal implants during RT applied in bone metastases. The rate of artefacts compared to gold markers was found to be significantly lower, when PEEK was used during CT stimulation for RT planning for image-guided radiotherapy (IGRT) applications in a study by Shen et al. [3]. Therefore, PEEK markers are recommended for use in liver-directed IGRT.

Currently various firms have added PEEKs to their list of fiducial markers used for IGRT, and it has been presented as another alternative to the use of gold markers. The PEEK markers are ready to be used as gold markers, particularly in prostate RT.

On the other hand, there is a disadvantage of PEEK use, which has not been described in the literature. In our practice, we place three gold markers in the prostate prior to RT in patients in whom a definitive RT directed to the prostate is planned. Therefore, kV images, rather than cone beam CT, are used during the treatment and the patient is exposed to less radiation and the duration of stay in the treatment device is shortened. The patients undergo CT simulation for RT planning following the placement of gold markers in the prostate, and the CT images obtained are evaluated on the treatment planning system (TPS). However, the CT artefacts produced by the gold disrupt the dose distribution (Fig. 1). Emerging artefacts are straightened by various applications on the TPS; however, this condition causes uncertainty 


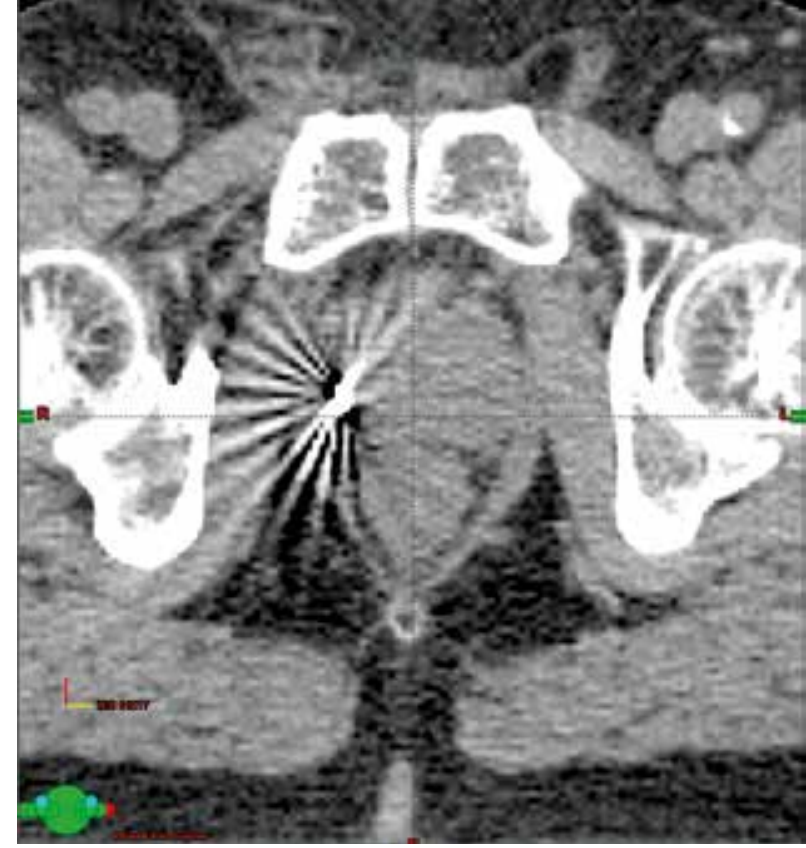

Fig. 1. Treatment planning system, axial CT view, gold marker, and CT artefacts

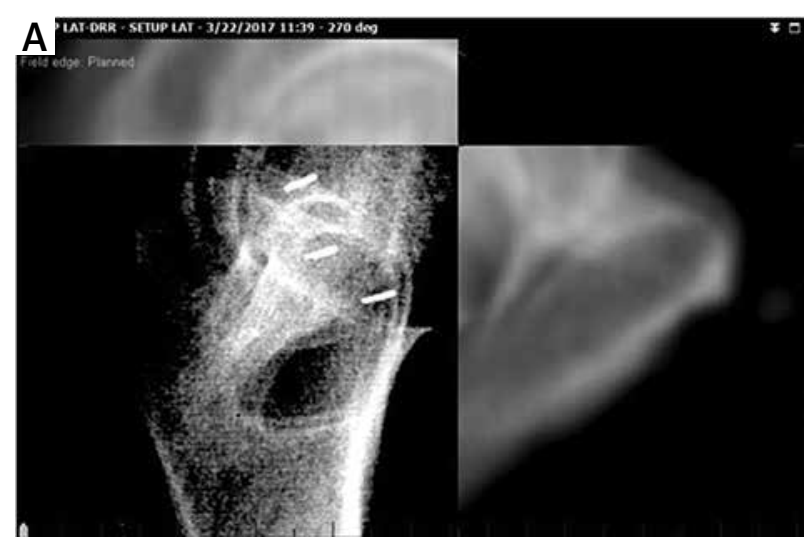

Fig. 3. Treatment device, gold markers, AP and lateral kV images
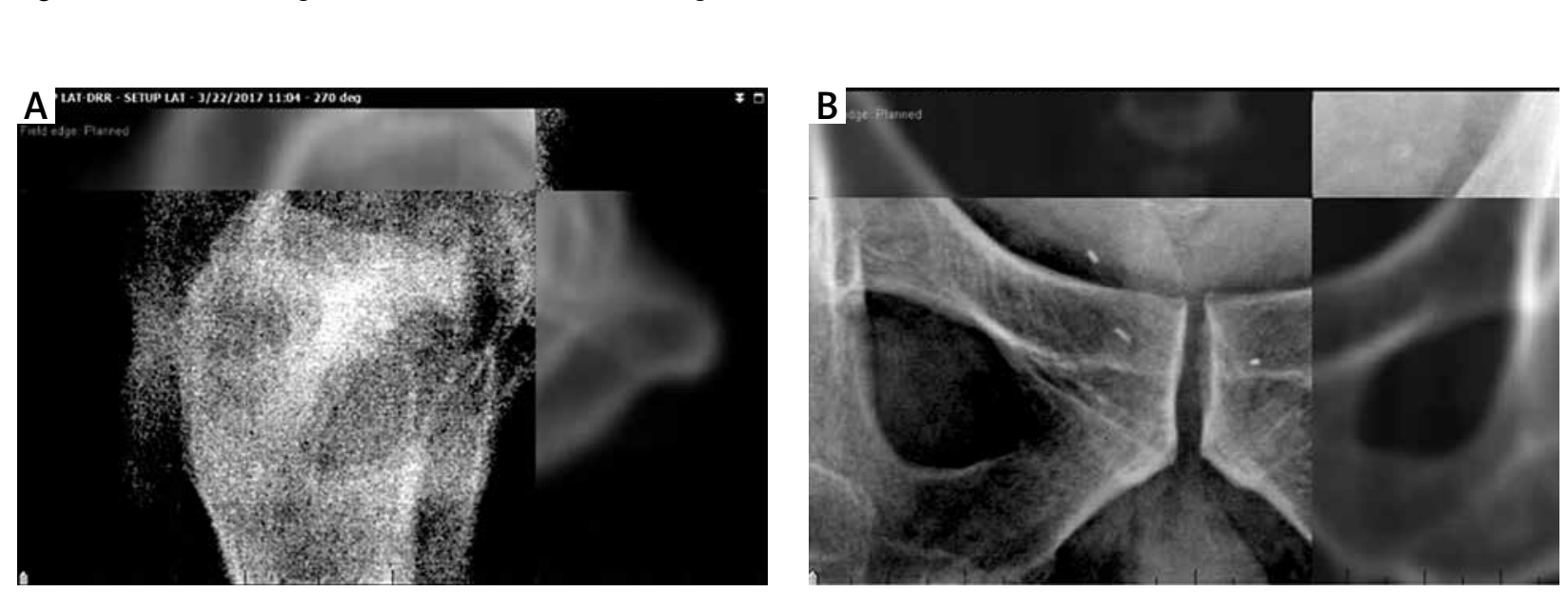

Fig. 4. Treatment device, PEEK markers, AP and lateral $k V$ images (PEEK markers could not be seen in the lateral image)

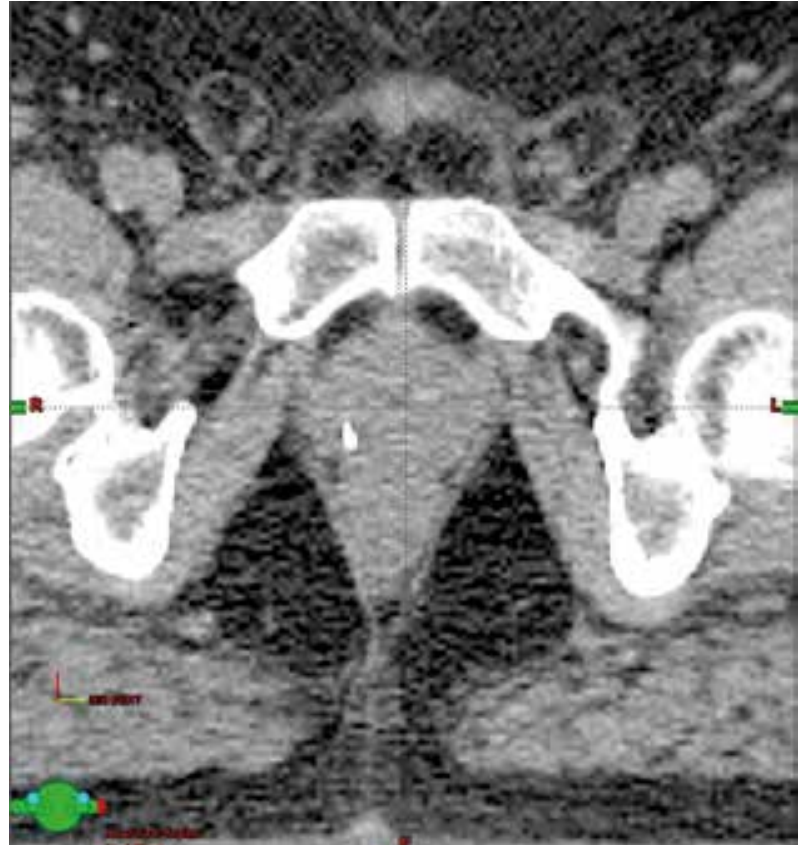

Fig. 2. Treatment planning system, axial CT image, PEEK marker, and no $\mathrm{CT}$ artefacts

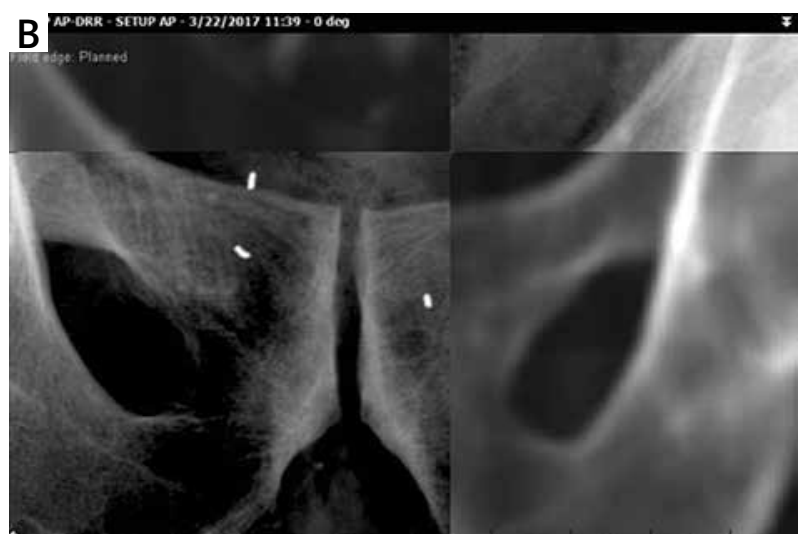



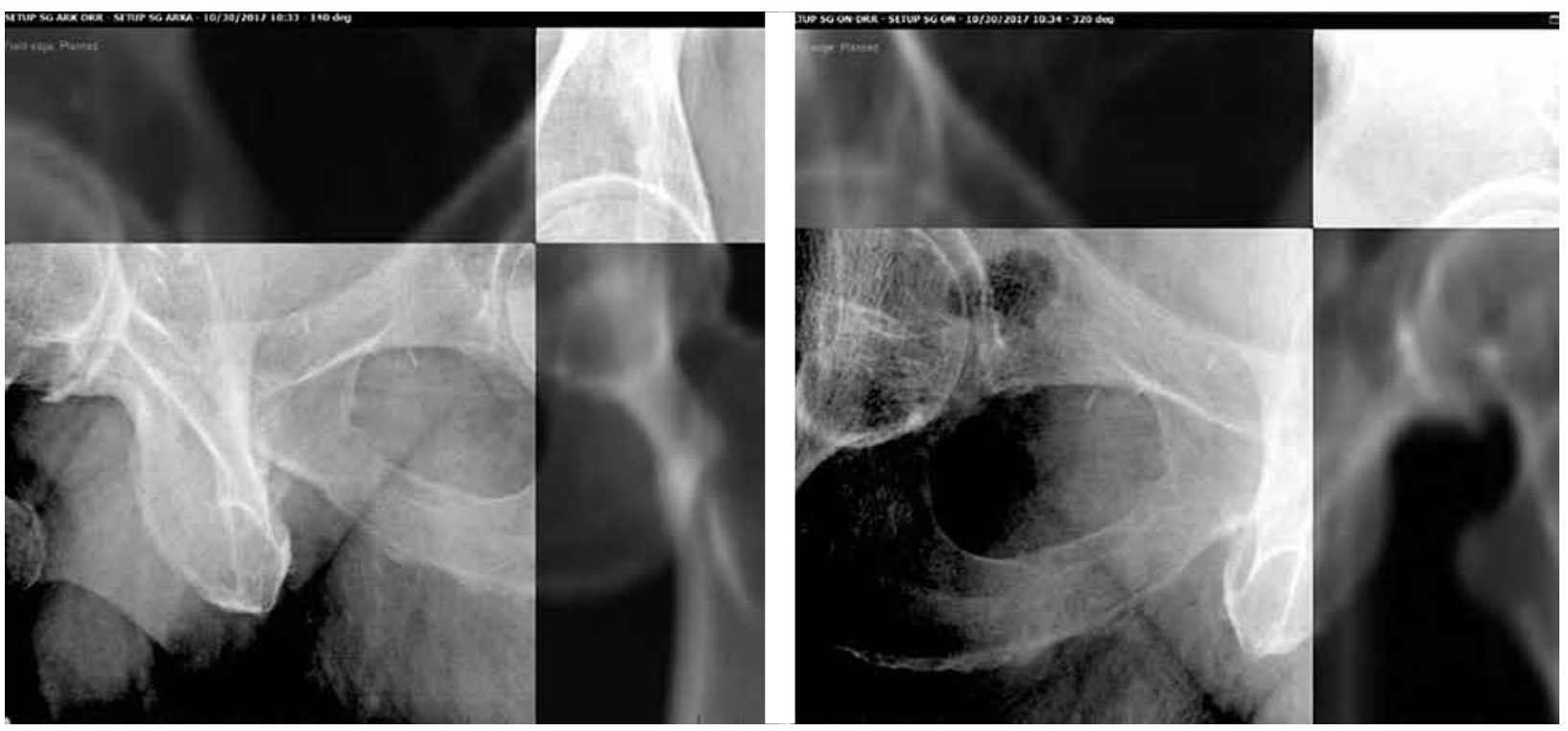

Fig. 5. Treatment device, PEEK markers, oblique kV images ( 140 degrees and 320 degrees)

in the dose distribution and prolongs the time for planning.

We have started to use PEEK, rather than gold, in prostate RT for a while to reduce artefacts. CT images of the PEEK yielded a very low level of artefacts on TPS without any correction on TPS (Fig. 2). However, no clear evaluation could be performed due to superposition of the femurs and PEEK during the treatment in AP and lateral kV port images and lateral $\mathrm{kV}$ images. This situation was not seen with the use of gold markers (Figs. 3-4). This can be attributed to the fact that the density of PEEK was lower than that of the gold, and it could not be defined among the bony structures. The most practical solution for this problem was to perform an oblique $\mathrm{kV}$ evaluation instead of lateral $\mathrm{kV}$ evaluation (Fig. 5). Hence, this disadvantage could have been overcome and the advantages provided by the PEEK application might have been beneficial.

Lateral $k V$ evaluation in prostate RT using a PEEK marker is inappropriate due to the low density of PEEK, compared to the gold, and superposition of the PEEK with the bones. However, the PEEK markers can be easily used in the RT directed to non-prostate targets that are not surrounded by bony structures. For prostate RT, on the other hand, the evaluation can be performed by oblique kV images, rather than discontinuing the use of PEEK markers. Nonetheless, further studies with larger patient series are needed to conclude.

The author declares no conflict of interest.

\section{References}

1. Panayotov IV, Orti V, Cuisinier F, et al. Polyetheretherketone (PEEK) for medical applications. J Mater Sci Mater Med, 2016; 27: 118.

2. Nevelsky A, Borzov E, Daniel S, et al. Perturbation effects of the carbon fiber-PEEK screws on radiotherapy dose distribution. J Appl Clin Med Phys 2017; 18: 62-68.
3. Shen S, Jacob R, Popple R, et al. SU-E-J-38: Improved DRR Image Quality Using Polyetheretherketone (PEEK) Fiducial in Image Guided Radiotherapy (IGRT). Medical Physics 2015; 42: 3272.

\section{Address for correspondence}

\section{Cenk Ahmet Sen}

Department of Radiation Oncology

Izmir Medical Park Hospital

1825 Sok. No:12 Yenigirne Bulv

35220 Izmir, Turkey

e-mail: cenkasen@gmail.com

Submitted: 26.02 .2018

Accepted: 2.03.2018 